\title{
Modeling the Spread of Ebola with SEIR and Optimal Control
}

\author{
Harout Boujakjian \\ Faculty Advisor: Tim Sauer
}

June 27, 2016

\section{Abstract}

Ebola is a virus that causes a highly virulent infectious disease that has plagued Western Africa, impacting Liberia, Sierra Leone, and Guinea heavily in 2014. Understanding the spread and containment of this disease is vital to its containment and eventual elimination. We use an SEIR model to simulate the transmission of the disease. The model is validated with data from the World Health Organization. Optimal control theory is used to explore the effect of vaccination and quarantine rates on the SEIR model. The goal is to explore the use of these control strategies to effectively contain the Ebola virus.

\section{Introduction}

Ebola was first identified in Congo in 1976. Its high fatality rate has made it one of the world's most feared diseases. Although the original strain (Ebola Zaire) in Congo was contained, other cases have popped up over the years. Several strains have been identified in nearby African countries. In 2014, Ebola once again reemerged in Guinea. It spread to neighboring countries Sierra Leone and Liberia, and there have been over 7,000 collective deaths in the three countries. In 2014, the same strain emerged in Liberia, Sierra Leone, and Guinea that was present in Congo in 1976 [13]. Without intelligently planned intervention, the disease spreads rapidly, ravaging cities along the way. Optimal intervention strategies are crucial to the containment of Ebola. Quarantine, proper handling of dead bodies, and experimental vaccination are three intervention strategies.

In this article, we use a generalized SIR (susceptible-infected-recovered) model to simulate the transmission of Ebola. Using SIR models is one way to further enhance our comprehension of disease transmission. These models are dynamical systems that compartmentalize a population into sub-populations of people who are susceptible to sickness, have a latent form of a disease, recovering from an infection, etc. Quantifying disease dynamics allows us to apply sophisticated mathematical tools that can shed light on disease transmission. Previous work for modeling the dynamics of Ebola include the 2004 article by Chowell et al. [4], and many papers specific to the 2014 outbreak $[1,2,3,5,9,12,15,19,22,21]$. 
SIR models were devised by A.G. McKendrick and W.O. Kermack in 1927 [8]. The basic model

$$
\begin{aligned}
\dot{S} & =-\beta I \frac{S}{N} \\
\dot{I} & =\beta I \frac{S}{N}-\mu I \\
\dot{R} & =\mu I
\end{aligned}
$$

is a three compartment nonlinear system of differential equations with two fundamental parameters: transmission rate and recovery rate. It portrays the dynamics of an infected sub-population where the individuals have the ability to spread a contagious disease. The variable $\mathrm{S}$ represents the susceptible population, which are those individuals who can contract the disease. The infected subpopulation is represented by the variable I, and those who survive through the infectious period are moved into the recovered compartment $\mathrm{R}$. In the simplified model, $\beta$ and $\mu$ are the respective transmitting and recovery rates

It is important to note that this is an idealized model. The population is assumed to be homogeneous, recovered sub-populations remain immune once they recover from the disease, and there is no birth or death. However, these models come in many shapes and flavors. By adding certain parameters, the models can include specific characteristics that are fundamental to a disease. The basic SIR model can be generalized [6]. Each of these compartmental models focus on certain sub-populations. Parameters that are commonly used include birth and death rates, in addition to interaction rates between compartments.

We use the SEIR model proposed by Chowell et al. [4]. The additional compartment E represents the exposed individuals who are in the incubation period.

$$
\begin{aligned}
\dot{S} & =-\beta I \frac{S}{N} \\
\dot{E} & =\beta I \frac{S}{N}-k E \\
\dot{I} & =k E-\gamma I \\
\dot{R} & =\gamma I
\end{aligned}
$$

The parameters $\beta, \gamma$, and $\mathrm{k}$ represent the transmitting rate, recovery rate, and incubation rate, respectively. The difference between the exposed and infected is that the former have contracted the disease but are not infectious, and the latter can spread the disease. In [4], SEIR is used to model the Ebola breakouts, and data from two documented cases in Congo in 1995 and Uganda in 2000 are used. Rigorous statistical analysis is used to determine the transmitting rate and recovery rate. The focus of the paper was on the reproduction number $R_{0}$, which is the average number of secondary cases generated by an infected individual [6].

Our modifications of the model derived by [4] include adding two parameters: vaccination and quarantine. The modifications portray the strategies used by countries to eliminate the disease. The World Health Organization (WHO) provided data on the 2014 Ebola outbreak in Liberia, Sierra Leone, and Guinea. Using the data from the WHO, we determine the parameters $\beta$ and $\mu$.

Optimal control is a powerful optimization technique used to derive the best control strategies. A set of differential equations are used with specific control rates that minimize an objective functional, which include all the variables that will be minimized. Pontryagin's maximum principle validates the existence of an optimal control [11]. This theory provides the mathematical groundwork for the use of optimal control in dynamical systems. 
Optimal control is applied to the SEIR. The two controls are vaccination and quarantine. There is currently no extensively used vaccine, although there has been talk of an experimental vaccine that seems promising [16]. Quarantine is currently a procedure that is heavily utilized in order to contain infected individuals. Both of these controls are contrasted and analyzed over time. We use the forward-backward sweep method prescribed by Lenhart [11] to obtain approximate numerical solutions. Thus, we include graphs of each of these controls with multiple arbitrary monetary values. This allows us to demonstrate the effects that price have on the amount of control applied. We notice that the implementation strategy over time can have counter-intuitive solutions depending on the duration of intervention.

In section 1, the basic details of the SEIR model applied to the 2014 Ebola outbreak data are introduced. Section 2 covers the fundamental ideas of optimal control, and discusses the numerical implementation of control applied to our model. The results of our analysis are described in section 3 , and section 4 contains a discussion of the results and their limitations.

\section{SEIR Model}

In this section, we formulate a four component SEIR (Susceptible-Exposed-Infected-Recovered) model that contains both vaccination and quarantine as ways to decrease the susceptible and infected.

$$
\begin{aligned}
\dot{S} & =-\beta I \frac{S}{N}-v S \\
\dot{E} & =\beta I \frac{S}{N}-\sigma E \\
\dot{I} & =\sigma E-\mu I-q I \\
\dot{R} & =\mu I+v S+q I
\end{aligned}
$$

$$
\begin{array}{ll}
\beta=\text { transmitting rate } & v=\text { vaccination } \\
\mu=\text { recovery rate } & q=\text { quarantine } \\
\sigma=\text { incubation rate } & \mathrm{N}=\text { total population }
\end{array}
$$

The interaction term $\beta I \frac{S}{N}$ is crucial to the model. A certain amount of interactions, dependent on the transmission rate $\beta$, between a susceptible and an infected will result in the susceptible contracting a latent form of Ebola. Thus, that susceptible will move into the exposed class. However, a certain amount of susceptibles will be vaccinated $v S$ and move straight into the recovered class without becoming infected. The exposed compartment then decreases at some rate $\sigma$, whose latent form of the virus now becomes infectious. Similarly, some of the infected $\mu I$ will recover without treatment and move into the recovered class.

One equilibrium exists for this model, the disease free equilibrium. The disease free equilibrium, where there are no infected or $I=0$, is $E_{0}=(0,0,0,0)$. Since there is no birth rate in this system, it is a trivial equilibrium.

In order to verify that the SEIR model accurately depicts the state of the disease, we use data provided by the World Health Organization [24] and test our model against the actual data. A fifth compartment is added to the SEIR, $\dot{C}=\sigma E$. The inclusion of $C$ does not disturb the dynamics of the SEIR; it simply sums the exposed population. We fit the model to data from three countries: Liberia, Sierra Leone, and Guinea. To determine the best fit, a range of $\beta$ and $\mu$ values are evaluated and the curve with the smallest root-mean-square error (RMSE) is selected. The incubation rate $\sigma$ used is 0.2 , which is 5 days [4]. The three curves are shown in Figure 1. 


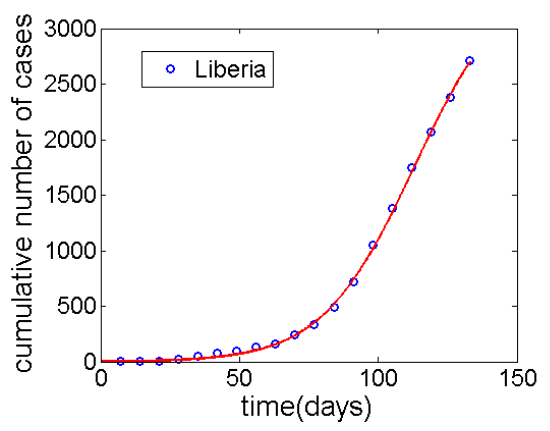

(a)

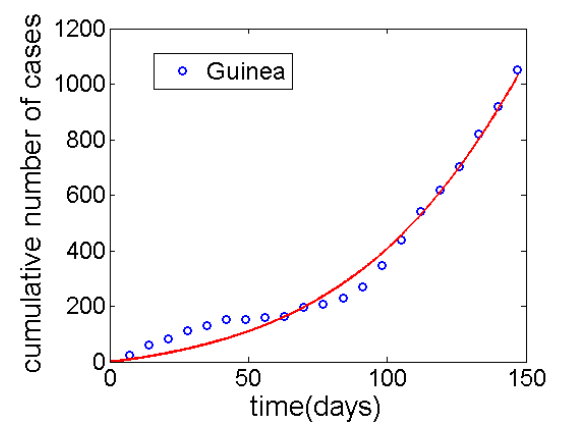

(b)

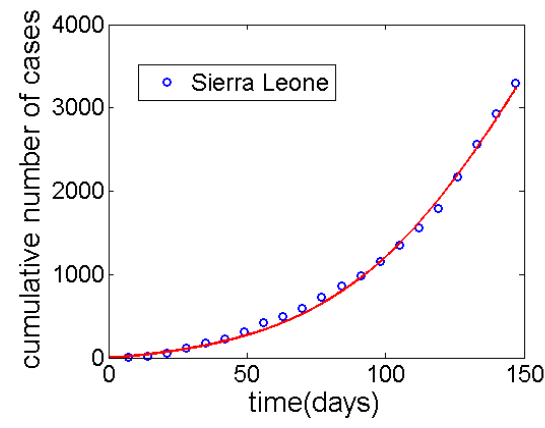

(c)

Figure 1: The graphs display time versus the cumulative number of infections for the three countries studied. The blue dots represent weekly amounts which are obtained by the WHO. The red curves denote the cumulative number of cases $C(t)$. The initials conditions are as follows: (a) Liberia: $S_{0}=4800, E_{0}=$ $1, I_{0}=1, R_{0}=0$ (b) Guinea: $S_{0}=12500, E_{0}=1, I_{0}=12, R_{0}=0$ (c) Sierra Leone: $S_{0}=19000, E_{0}=$ $2, I_{0}=21, R_{0}=0$.

We use different time spans for the countries in order to include the time leading up to the peak infectious period. The time intervals used correspond to when the countries had the most Ebola cases covered by the weekly situation reports released by the WHO. The dates used from 2014 were as follows: Liberia: June 1 - October 5, Sierra Leone: May 25 - October 12, Guinea: May 25 October 12. Since we are fitting the uncontrolled model, it is important to use the data for the spread of Ebola prior to major effective intervention. Therefore, our fitting only uses data before the peak of the infectious period.

The reproduction number $R_{0}$ is also calculated for each of the three countries. For our purposes, $R_{0}=\beta / \mu$ suffices even though this is the simplified $R_{0}$ for the SIR model (1) presented in the introduction. There are more sophisticated ways to compute $R_{0}$, for example refer to [23]. Our calculated reproduction numbers, along with corresponding $\beta$ and $\mu$, are in Table 1 . Viewing $R_{0}$ as a threshold parameter, we can confirm that the disease will continue to spread for all three countries since $R_{0}>1$.

\begin{tabular}{|c|c|c|c|}
\hline & $\beta$ & $\mu$ & $R_{0}$ \\
\hline Sierra Leone & 0.27 & 0.21 & 1.29 \\
\hline Liberia & 0.29 & 0.16 & 1.81 \\
\hline Guinea & 0.20 & 0.16 & 1.25 \\
\hline
\end{tabular}

Table 1: The calculated $\beta, \mu$, and $R_{0}$ values for Liberia, Sierra Leone, and Guinea data fittings.

\section{Optimal Control}

Many previous researchers have contributed to understanding how to introduce control into various types of SIR models. In [14], Neilan and Lenhart make a six compartment model for cholera. There are four compartments for the human population, and two compartments for the bacteria vector. They have three controls: vaccination, antibiotics and rehydration, and environmental sanitation.

Some previous work has discussed optimal control applied to general disease models. In [7], Kar and Batabyal construct an SIR model with a logistic growth rate. The model has a separate 
death rate in the recovered compartment for those individuals who die and leave the population. They apply optimal control to the vaccination rate, using the same techniques listed in [11]. In [10], Ledzewicz and Schattler analyze an SIR model with vaccination and treatment. They apply optimal control to it and analyze the structure of singular controls.

Intervention strategies are crucial to stopping the spread of a disease. For contagious diseases like Ebola, effectively containing the disease could save thousands of lives. Due to the complexity of interventions in a highly infectious disease, it is critical to apply them in the most cost-effective way. To determine the optimal amount of control, we use Pontryagin's Maximum Principle to derive an optimality condition. This condition is derived from the Hamiltonian and is a function of both the SEIR and adjoint equations.

Our approach to optimal control of Ebola will be to apply vaccination and quarantine controls to the SEIR model (3). Pontryagin's Maximum Principle lays the mathematical groundwork for introducing a control into a dynamical system [18].

We will consider the finite-dimensional optimal control problem. In order to formulate an optimal control problem, a state and objective functional are required. Consider the following objective functional $J(u)$ subject to some differential equation (state)

$$
\begin{aligned}
J(u) & =\int_{t_{0}}^{t_{1}} f(t, x(t), u(t)) d t \\
\dot{x} & =g(t, x(t), u(t)) \\
x\left(t_{0}\right) & =x_{0}
\end{aligned}
$$

and let $u$ be a control strategy for the differential equation. We assume that $f(t, x(t), u(t))$ and $g(t, x(t), u(t))$ are continuously differentiable functions in their arguments and concave in the state $\mathrm{x}(\mathrm{t})$ and control $\mathrm{u}(\mathrm{t})$. Suppose $u^{*}$ and $x^{*}(t)$ are optimal for the given objective functional and state equation. We define the adjoint variable $\lambda(t)$ as a piecewise differentiable function. Theorem 1.3 in [11] states that for the optimal control and associated state, there exists a piecewise differentiable $\lambda(t)$ such that

$$
H\left(t, x^{*}(t), u(t), \lambda(t)\right) \leq H\left(t, x^{*}(t), u^{*}(t), \lambda(t)\right)
$$

for every control out of a set of permissible controls for $u$ at each time $t$, where the Hamiltonian $\mathrm{H}$ is defined as

$$
H=f(t, x(t), u(t))+\lambda(t) g(t, x(t), u(t))
$$

and

$$
\begin{aligned}
& \lambda^{\prime}(t)=-\frac{\partial H\left(t, x^{*}(t), u^{*}(t), \lambda(t)\right)}{\partial x} \\
& \lambda\left(t_{1}\right)=0
\end{aligned}
$$

Lastly, setting the partial of $\mathrm{H}$ with respect to $\mathrm{u}$ equal to zero and solving for $\mathrm{u}$ results in the optimal control.

We use the SEIR subpopulations as the state variables. The objective functional will focus on minimizing three variables: the infected population, vaccination rate, and quarantine rate. We define the objective functional as

$$
\min _{v, q} J(v, q)=\min _{v, q} \int_{t_{0}}^{t_{1}}\left[I(t)+\frac{A}{2} v^{2}(t)+\frac{B}{2} q^{2}(t)\right] d t
$$

We treat the analysis of the control variables separately. When we explore the vaccination rate curves in depth, we set $q=0$ in the SEIR model. Effectively, we are "shutting off" the quarantine 
intervention and analyzing the system with only vaccination. Similarly, we set $v=0$ when we want to investigate quarantine. The model is set up so that letting a control parameter equal to zero does not disturb the dynamics of the rest of the compartments.

In order to find an optimal solution, we derive the Hamiltonian, defined as the integrand of the objective functional plus the adjoint variable $\lambda(t)$ multiplied by right hand side of the ODE. In this case, we add the integrand of the objective functional with the dot product of the vector $\lambda(t)$ and the four-dimensional state of the SEIR model, so the Hamiltonian is

$$
\begin{aligned}
H \equiv I(t) & +\frac{A}{2} v^{2}(t)+\frac{B}{2} q^{2}(t)+\lambda_{S}\left(-\beta I \frac{S}{N}-v S\right) \\
& +\lambda_{E}\left(\beta I \frac{S}{N}-\sigma E\right)+\lambda_{I}(\sigma E-\mu I-q I) \\
& +\lambda_{R}(\mu I+v S+q I) .
\end{aligned}
$$

Using the Hamiltonian, we derive the adjoint equations. Since the ODE system has four equations, there will be one adjoint equation for each of the S, E, I, and R compartments. The adjoint equation for $\mathrm{S}$ is defined by setting the time derivative of the adjoint variable equal to $-\frac{\partial H}{\partial S}$. The other adjoints are defined similarly

$$
\begin{aligned}
& \dot{\lambda_{S}}=\lambda_{S}\left(\beta \frac{I}{N}+v\right)-\lambda_{E} \beta \frac{I}{N}-\lambda_{R} v \\
& \dot{\lambda_{E}}=\lambda_{E} \sigma-\lambda_{I} \sigma \\
& \dot{\lambda_{I}}=-1+\lambda_{S} \beta \frac{S}{N}-\lambda_{E} \beta \frac{S}{N}+\lambda_{I}(\mu+q)-\lambda_{R}(\mu+q) \\
& \dot{\lambda_{R}}=0
\end{aligned}
$$

Here we use Pontryagin's Maximum Principle, which states that the Hamiltonian with the optimal control will be greater than or equal to the Hamiltonian with any other control out of a set of permissible controls [18]. In order to determine the optimal controls, we derive the optimality conditions by setting $\frac{\partial H}{\partial v}=0$. Solving for v yields

$$
v^{*}=\frac{\lambda_{S} S-\lambda_{R} S}{A}
$$

We can solve for q similarly

$$
q^{*}=\frac{\lambda_{I} I-\lambda_{R} I}{B}
$$

A numerical method called the forward-backward sweep [11] is used to determine the optimal vaccination and quarantine rate. This method solves the SEIR equations (3) forward in time from $t_{0}$ to $t_{1}$ using a fourth order Runge-Kutta (RK4) method. The RK4 code used was from [20]. The initial conditions selected were arbitrary since it is difficult to determine exact numerical values. We used initial conditions that were similar to the ones used for the fittings: $S_{0}=4800, E_{0}=10$, $I_{0}=10, R_{0}=0$. The time step is $\Delta t=0.2$, and the unit of time for the model is in days.

Next, the forward-backward sweep solves the adjoint equations (10) backward in time from $t_{1}$ to $t_{0}$. This means using negative step size, starting from the end and moving backwards. In our case, we used RK4 again with $\Delta t=-0.2$ as the step size. The optimality conditions are updated after each iteration using the values from SEIR and adjoint equations.

The following is a general layout of the steps for the forward-backward sweep. Denote $\vec{x}=$ $[S(t), E(t), I(t), R(t)]^{T}, \vec{\lambda}=\left[\lambda_{S}(t), \lambda_{I}(t), \lambda_{E}(t), \lambda_{R}(t)\right]^{T}$, and $\vec{u}=[v(t), q(t)]^{T}$. 
1. Make an initial guess for control $\vec{u}$.

2. Use initial condition for $\vec{x}$ and controls to solve $\vec{x}$ forward in time from $t_{0}$ to $t_{1}$.

3. Use $\vec{x}$ and $\vec{u}$ to solve $\vec{\lambda}$ backward in time from $t_{1}$ to $t_{0}$ with transversality condition $\vec{\lambda}\left(t_{1}\right)=0$ as the initial conditions for the adjoint.

4. Update control $\vec{u}$ with optimality condition, which uses the new $\vec{x}$ and $\vec{\lambda}$ values

5. Iterate steps 2 through 4 until convergence is reached.

One iteration involves a full forward and backward sweep from $t_{0}$ to $t_{1}$ and back to $t_{0}$ followed by updating the optimality condition (11) or (12). On average, convergence was reached within about 15 to 20 iterations.

\section{Results}

We use Matlab to run the forward-backward sweep on the SEIR model with each control separately. Figure 2 shows the resulting populations for the Susceptible, Exposed, Infected, and Recovered compartments, respectively. The blue lines represent no intervention strategy, which is just the disease running its course through the population and eventually dying out. The disease dies out because there is no birth rate variable in the model. The red curves indicate each compartment with an optimal intervention strategy, which reduces the amount of susceptible, exposed, and infected in the population. As a result of the vaccination the recovered increases dramatically. The quarantine helps reduce the infected population.

The plots for the controls give us the most information about how to implement any sort of intervention strategy. Vaccination and quarantine are two controls that help to contain the spread of the disease. Although there is currently no vaccine available for wide use, extensive research is being done to discover one [16]. Since no cost is available, we test the model with several arbitrary price values for the vaccine. This will allow us to make predictions about how a vaccine should be implemented once one is readily available for use. A similar analysis is done for quarantine. The plots for these curves contain multiple values for A and B, which are the proportionality parameters in the Hamiltonian. They can be thought of as weights and only allow amounts of control that are within the specified range.

The graphs in Figure 3 are the vaccination and quarantine rates as a function of time over two intervals: 100 days and 300 days. These time intervals designate the amount of days that the control is being implemented in the population. The vaccination curves seem to follow a steady pattern. As the price of the vaccine increases, the vaccination rate decreases. We believe that the vaccination rate is highest at the beginning because the susceptible sub-population is the largest at day 0. Most of the vaccines are given out immediately. This move the vaccinated individuals straight to the recovered sub-population, avoiding any infection at all. After implementing the vaccine for about 20 days, the susceptible population decreases significantly, which means that there isn't a need to vaccinate as much. Thus, the vaccination rate decreases significantly too. This holds in both the 100 day and 300 day cases. The time interval does not seem to change the way the intervention will be implemented.

However, quarantine doesn't adhere to the same intuitive pattern. For 100 days, the quarantine rate decreases as the price of quarantining increases. Unlike the vaccination rate curves, there is a steady amount of quarantine intervention all the way until day 100. We notice that the curves are 


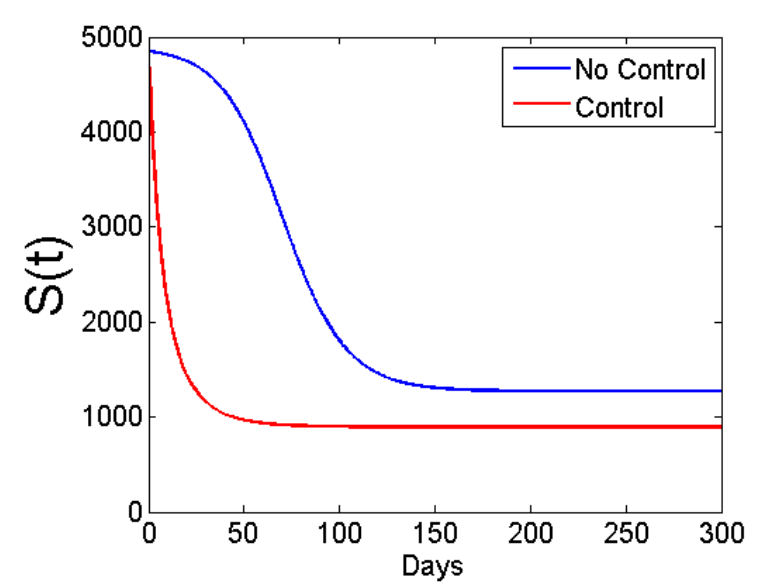

(a)

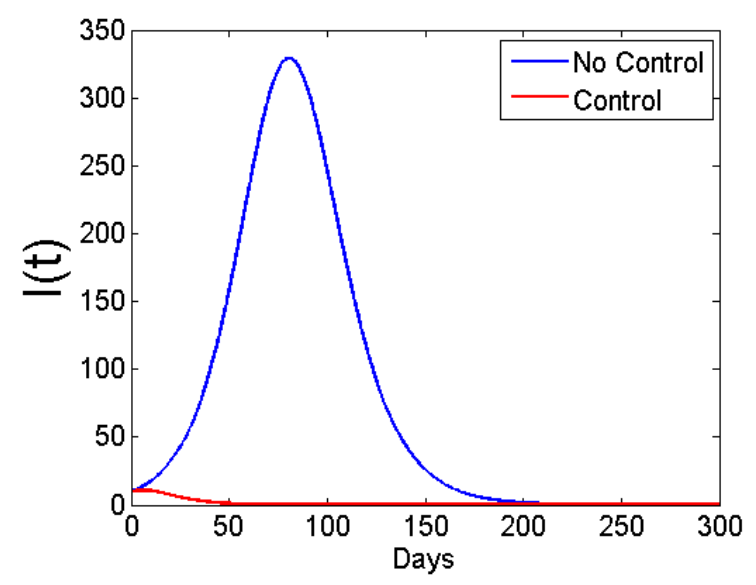

(c)

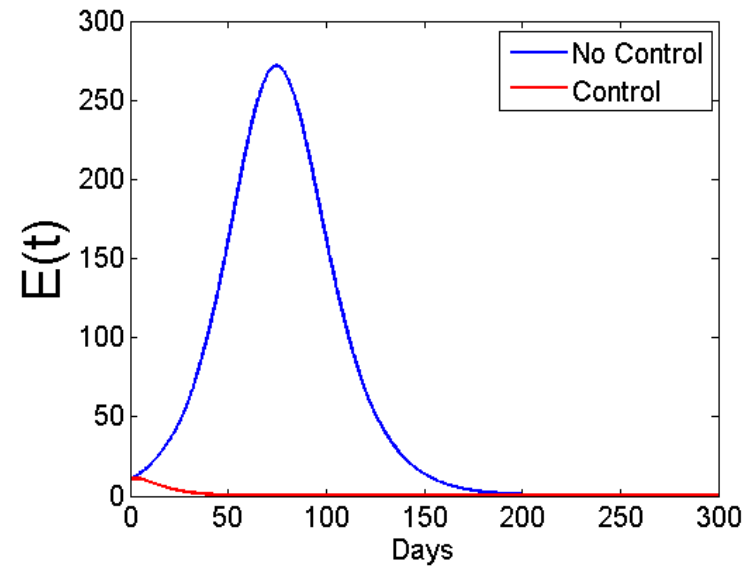

(b)

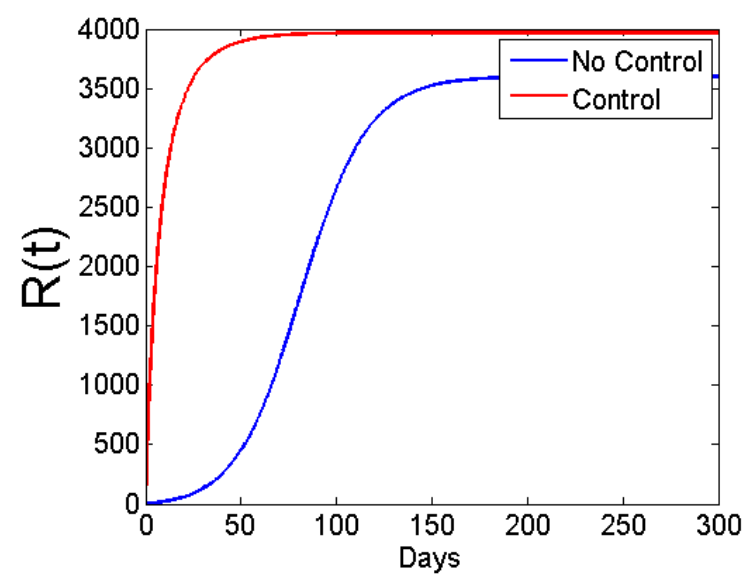

(d)

Figure 2: The four plots represent each of the curves for SEIR. The blue curves are the disease running its course through the population. The red curves display the effects of intervention on containing the spread of the disease. The initial conditions for the system are $S_{0}=4850, E_{0}=10, I_{0}=10, R_{0}=0$. The parameter values are $\beta=0.29, \mu=0.16, \sigma=0.2, \mathrm{~A}=2900, \mathrm{~B}=1500$.

convex. In contrast, most of the vaccination runs out by day 80 . The quarantine curves for 300 days are more interesting. As the price of quarantine increases, there is a more complicated relationship to the quarantine cost. It seems that each price has a separate optimal quarantine strategy. For the more expensive quarantine rates, they seem to have a shape that is similar to the size of the infected population. This may be because as the infected population increases, the quarantine rate also increases to combat the infections and move the individuals to the recovered compartment.

\section{Discussion}

The goal of this paper is to explore intervention strategies to limit the transmission of the Ebola virus using an SEIR model and optimal control. Compared to basic SIR models, adding the exposed compartment allows there to be an incubation period. This could be viewed as a time delay in the model. The WHO provided data on the weekly cases of Ebola in three countries. We fit the model 


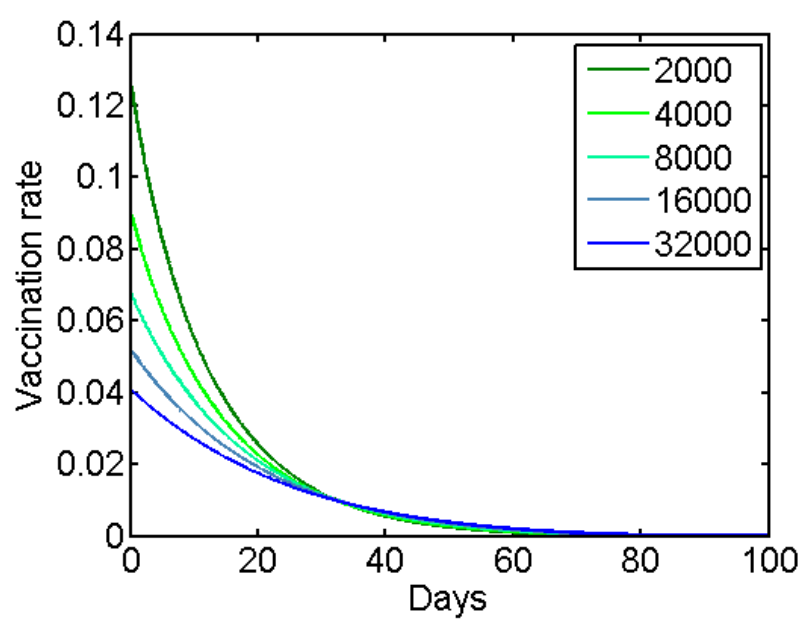

(a)

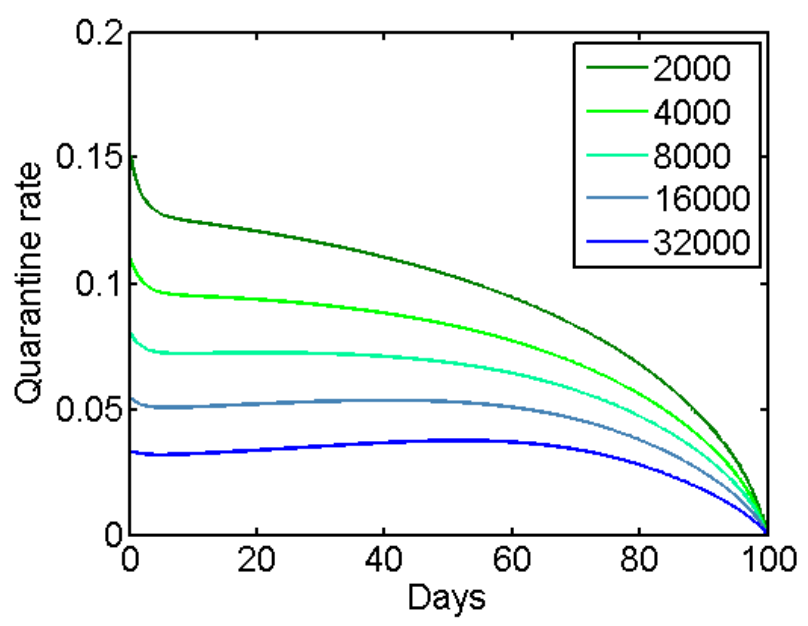

(c)

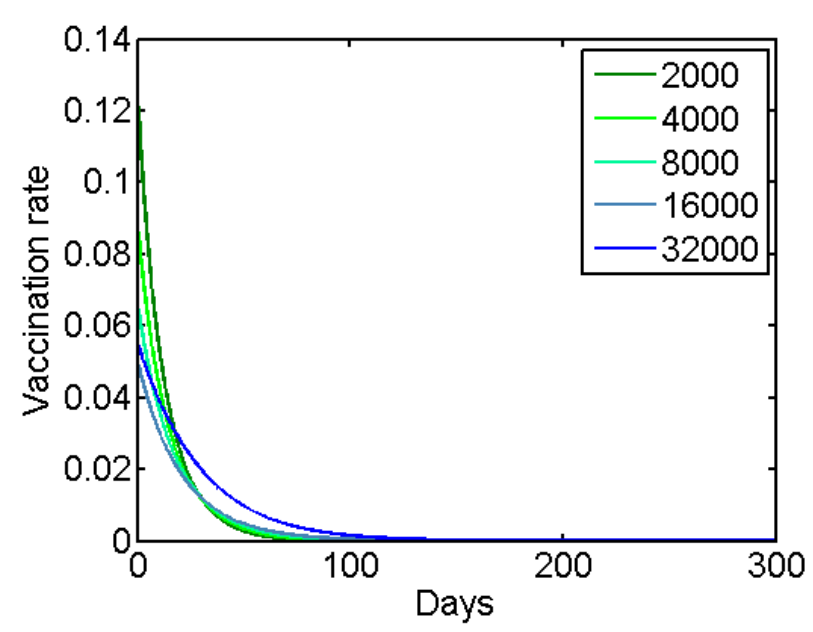

(b)

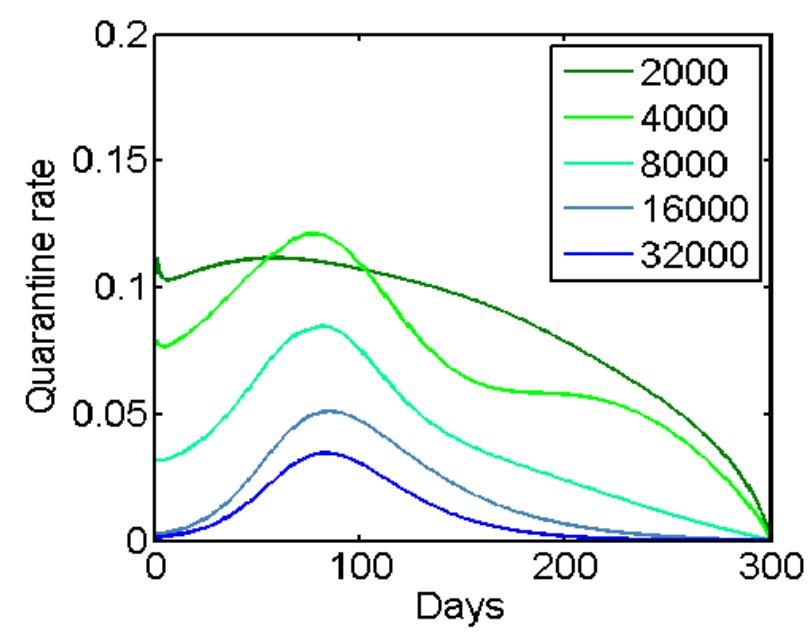

(d)

Figure 3: Plots of each of the controls over time. The initial conditions for the system are $S_{0}=4850, E_{0}$ $=10, I_{0}=10, R_{0}=0$. The parameter values are $\beta=0.29, \mu=0.16, \sigma=0.2$. The values for the costs $\mathrm{A}$ and $\mathrm{B}$ are 2000, 4000, 8000, 16000, 32000.

to the data and determined parameters that made the approximations the most accurate. The reproduction number was calculated based on the $\beta$ and $\mu$ values determined from the fittings.

We implemented optimal control in the SEIR model using a Hamiltonian formulation. A derivation of the Hamiltonian, adjoint equations, and optimality conditions are provided. Using a numerical method known as the forward-backward sweep, we simulated outcomes of the SEIR equations with and without control. It is evident that introducing an optimal control is an effective measure of decreasing the infected in the population. Our focus was on two intervention strategies: vaccination and quarantine. Graphs of the controls over time with a range of arbitrary cost parameters portrayed diverse results, allowing us to compare the optimal strategies. Vaccination rates increase as the price decreases and most is given out almost immediately. This is not the case for quarantine implementation. There was a regular amount of quarantine implemented during most of the intervention duration. However, when the intervention is introduced for longer time periods, such as 300 days, there is no simple description of the pattern that results. 
The calculations for the reproduction number were done using the $R_{0}$ from (1). A more thorough treatment of the reproduction number is provided by [23], which may more accurately account for the exposed compartment. Along with studying $R_{0}$, further explanation of the quarantine curves for 300 days would be welcome. This may reveal intervention strategies that are not customarily used.

Another way to expand our analysis would be to add spatial dimensions to the model. The WHO provides local data on Ebola, which shows the cumulative infections in many of the cities in Liberia, Sierra Leone, and Guinea. Developing a partial differential equation would shed light on how the disease spreads spatially. Also, adding other compartments to the model is another possibly to improve the approximations, such as an entire compartment for the quarantined or vaccinated individuals. Applying optimal control to variables in the different compartments may give results that are as unusual as the 300 day quarantine curves.

\section{Acknowledgments}

I would like to acknowledge my mentor, Dr. Tim Sauer, and the GMU EXTREEMS-QED program for help and support. I appreciate the comments from two reviewers whose advice greatly improved the manuscript. This work was partially supported by NSF grant DMS-1407087. 


\section{References}

[1] C. Althaus, Estimating the reproduction number of ebola virus (ebov) during the 2014 outbreak in west africa, PLoS currents, 6 (2014).

[2] C. Browne, H. Gulbudak, And G. WebB, Modeling Contact Tracing in Outbreaks with Application to Ebola, arXiv preprint arXiv:1505.03821, (2015).

[3] D. Chowell, C. Castillo-Chavez, S. Krishna, X. Qiu, and K. Anderson, Modelling the effect of early detection of Ebola, The Lancet Infectious Diseases, 15 (2015), pp. 148-149.

[4] G. Chowell, N. Hengartner, C. Castillo-Chavez, P. Fenimore, and J. Hyman, The basic reproductive number of Ebola and the effects of public health measures: the cases of Congo and Uganda, Journal of Theoretical Biology, 229 (2004), pp. 119-126.

[5] D. Fisman, E. Khoo, And A. Tuite, Early epidemic dynamics of the West African 2014 Ebola outbreak: estimates derived with a simple two-parameter model, PLoS currents, 6 (2014).

[6] H. Hethcote, The mathematics of infectious diseases, SIAM review, 42 (2000), pp. 599-653.

[7] T. Kar And A. Batabyal, Stability analysis and optimal control of an SIR epidemic model with vaccination, Biosystems, 104 (2011), pp. 127-135.

[8] W. Kermack And A. MCKendrick, A contribution to the mathematical theory of epidemics, in Proceedings of the Royal Society of London A: Mathematical, Physical and Engineering Sciences, vol. 115, The Royal Society, 1927, pp. 700-721.

[9] A. Kucharski and J. Edmunds, Case fatality rate for Ebola virus disease in west Africa, The Lancet, 384 (2014), p. 1260.

[10] U. LEDZEWICZ AND H. SCHÄTTLER, On optimal singular controls for a general SIR-model with vaccination and treatment, Discrete and Continuous Dynamical Systems, (2011), pp. 981990.

[11] S. Lenhart And J. Workman, Optimal control applied to biological models, CRC Press, 2007.

[12] J. Lewnard, M. Mbah, J. Alfaro-Murillo, F. Altice, L. Bawo, T. Nyenswah, AND A. Galvani, Dynamics and control of Ebola virus transmission in Montserrado, Liberia: a mathematical modelling analysis, The Lancet Infectious Diseases, 14 (2014), pp. 1189-1195.

[13] R. Martinez, Chronology of Ebola Virus Disease outbreaks, 19762014, 2014, http://healthintelligence.drupalgardens.com/content/ chronology-ebola-virus-disease-outbreaks-1976-2014.

[14] R. Neilan, E. Schaefer, H. Gaff, K. Fister, and S. Lenhart, Modeling optimal intervention strategies for cholera, Bulletin of mathematical biology, 72 (2010), pp. 2004-2018.

[15] H. Nishiura AND G. Chowell, Early transmission dynamics of Ebola virus disease (EVD), West Africa, March to August 2014, Euro Surveill, 19 (2014), p. 20894. 
[16] A. Oplinger, Experimental Ebola vaccine safe, prompts immune response, 2015, http://www.nih.gov/news-events/news-releases/ experimental-ebola-vaccine-safe-prompts-immune-response.

[17] H. PESch AND M. Plail, The cold war and the maximum principle of optimal control, Optimization Stories. Documenta Mathematica, (2012).

[18] L. Pontryagin, V. Baltyanski, R. Gamkrelidze, and E. Mischenko, The mathematical theory of optimal processes, VI. John Wiley \& Sons. I, (1962).

[19] C. Rivers, E. Lofgren, M. Marathe, S. Eubank, and B. Lewis, Modeling the impact of interventions on an epidemic of Ebola in Sierra Leone and Liberia, PLoS currents, 6 (2014).

[20] T. SAuer, Numerical Analysis, 2nd Edition, Pearson, 2011.

[21] J. Shaman, W. Yang, And S. Kandula, Inference and forecast of the current West African Ebola outbreak in Guinea, Sierra Leone and Liberia, PLoS currents, 6 (2014).

[22] S. Towers, O. Patterson-Lomba, and C. Castillo-Chavez, Temporal variations in the effective reproduction number of the 2014 West Africa Ebola outbreak, PLoS currents, 6 (2014).

[23] P. Van Den Driessche And J. Watmough, Reproduction numbers and sub-threshold endemic equilibria for compartmental models of disease transmission, Mathematical Biosciences, 180 (2002), pp. 29-48.

[24] W. H. O. (WHO), Ebola response roadmap situation report, 2015. 